\title{
Enquête alimentaire dans trois secteurs de la basse vallée du Rhône : Codolet, Tresques, Camargue. Consommation/autoconsommation
}

\author{
B. DESCAMPS ${ }^{1}$, F. GUILLET ${ }^{2}$
}

RÉSUMÉ Une étude de l'impact dosimétrique du site de Marcoule sur les habitants de Codolet, le village considéré comme le plus exposé, a été réalisée par l'IPSN en 1996. Dans la partie impact lié à l'ingestion l'auteur a conclu à la nécessité de mieux connaître les habitudes alimentaires des habitants, et plus précisément les habitudes d'autoconsommation (consommation d'aliments d'origine locale, de production personnelle, fournis ou achetés). En effet en absence des habitudes d'autoconsommation "réelles et actuelles " la consommation a été considérée par l'auteur comme totalement de l'autoconsommation. Une enquête alimentaire a donc été réalisée en mai 1998 dans le village de Codolet mais aussi dans le village de Tresques, considéré comme un village non exposé, et dans la zone inondée de la Camargue lors des crues d'octobre 1993 et de janvier 1994. L'enquête est relative à une vingtaine de foyers sélectionnés dans chacun des trois lieux et le questionnaire d'enquête est constitué de 14 feuillets. Pour cette enquête, réalisée pendant une semaine complète, on utilise une unité «fictive» tenant compte de la grande variabilité de la composition des foyers. Cette unité est nommée * unité de consommation, uc $»$ et elle s'exprime en g ou en cl par uc et par jour ( $\left.\mathrm{g} \mathrm{ou} \mathrm{cl} \mathrm{uc} \mathrm{uc}^{-1} \mathbf{j}^{-\mathbf{l}}\right)$. Elle est en général assez peu différente d'une unité plus usuelle exprimée en g ou en I par habitant et par jour $\left(g\right.$ ou $\left.\mathrm{cl} \mathrm{h}^{-1} \mathrm{j}^{-1}\right)$. Les treize principaux aliments autoconsommés dans les trois échantillons enquêtés sont (moyennes en $\mathrm{g}$ ou

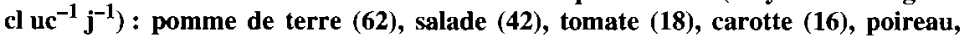
haricot vert et fraise (13), cerise et radis (11) pour les légumes et les fruits; oufs et poulet (15) pour les produits d'origine animale ; vin et eau (10) pour les liquides. Pour Codolet les valeurs d'autoconsommation des aliments pris en compte dans l'étude dosimétrique de 1996 sont toutes, à la seule exception du vin, très largement supérieures à celles relatives à la présente enquête. Une actualisation, avec nos valeurs, de cette étude dosimétrique aboutirait à une baisse très significative de la contribution de l'ingestion dans l'impact dosimétrique total.

ABSTRACT Food survey of three areas of the lower Rhone valley: Codolet, Tresques, and the Camargue. Consumption/home consumption.

In 1996 the IRSN studied the dosimetric impact of the Marcoule site on the inhabitants of reference village, Codolet. The ingestion-related impact highlighted by this study revealed the need for further familiarisation with the eating habits of the villagers, and more precisely their home consumption habits (that is

\footnotetext{
1 IRSN, Département de protection de l'environnement, B.P. 3, 13115 St Paul-les-Durance Cedex, France.

2 J.C.A. Consultants, 06640 St Jeannet, France
} 
consumption of locally-produced foodstuffs, own personal production, however supplied or purchased). Accordingly a food survey was carried out in May 1998 in the village of Codolet and also in the village of Tresques that acted as control village and in the area of the Camargue that was flooded when the Rhone burst its banks in October 1993 and January 1994. Knowledge of "real and current" home consumption habits is important as in the 1996 study, it was taken that all consumption was of the home consumption type. The survey, comprising a 14-page survey questionnaire, covers some twenty selected households per site. A "fictitious" unit was used to make allowance for the wide variations in household make-up for this type of weeklong survey. This unit is called a "unit of consumption, uc" and is expressed in $\mathrm{g}$ or cl per uc, per day ( $\mathrm{g}$ or $\mathrm{cl} \mathrm{uc}^{-1} \mathrm{j}^{-1}$ ). It differs very little from the more conventional unit expressed in $\mathrm{g}$ or $\mathrm{cl}$ per inhabitant, per day (g or $\mathrm{cl} \mathrm{h}^{-1} \mathrm{j}^{-1}$ ). The thirteen main home consumption foodstuffs in the three survey samples were (mean values in $\mathrm{g} \mathrm{or} \mathrm{cl} \mathrm{uc}^{-1} \mathrm{j}^{-1}$ ): potatoes (62), lettuces (42), tomatoes (18), carrots (16), leeks, French beans and strawberries (13), cherries and radishes (11) in the fruit and vegetable category; eggs and chicken (15) for produce of animal origin; wine and water (10) in the liquids category. As regards Codolet, the home consumption figures for foodstuffs included in the 1996 dosimetric study are all, with the exception of wine, much higher than those revealed by our food survey. Applying our figures to update this dosimetric study would cause the ingestion aspect of dosimetric impact to drop very significantly.

\section{Introduction}

Entre 1989 et 1996, l'IPSN/DPRE a effectué de nombreuses études radioécologiques autour du site de Marcoule dans les domaines aquatique et terrestre (Lambrechts et al., 1990, 1992, 1993, 1994, 1995, 1996 ; Lumia et Descamps, 1995 ; Descamps et Lumia, 1996 ; Descamps, 1997) dans le but de mettre en évidence un éventuel impact radioécologique des rejets liquides et gazeux des installations nucléaires présentes sur ce site nucléaire, avant l'arrêt de son activité « retraitement » fin 1997.

Une évaluation de l'impact dosimétrique du site, en particulier celui lié à l'ingestion ( $8 \mu \mathrm{Sv} \mathrm{an}^{-1}$ ), a été réalisée par I'IPSN (Després, 1996) à partir des données radioécologiques des domaines aquatique et terrestre de 1993. Cette étude, réalisée pour les habitants du village de Codolet, a mis en particulier en exergue la nécessité de connaître, avec le plus de précision possible, la qualité et la quantité des différents aliments consommés par les habitants. Une enquête alimentaire devait répondre à cette nécessité. Son intérêt résidait dans la substitution par des données locales, précises et actualisées, des données nationales ou au mieux régionales, globales et relativement anciennes, en matière de comportement alimentaire. Cette enquête a été réalisée en fin de printemps 1998 par Guillet de J.C.A. Consultants. Elle visait à qualifier et à quantifier la consommation et l'autoconsommation d'un certain nombre de familles; l'autoconsommation étant relative à la consommation d'aliments issus d'une production personnelle ou familiale (jardinage et élevage essentiellement) et/ou 
aux aliments achetés, d'origine locale avérée. Cette étude a porté sur des habitants de trois communes (Fig. 1) :

- Codolet, au sud immédiat de Marcoule, considéré comme le village le plus exposé aux rejets du site de Marcoule (le «village de référence » selon la définition de la Commission internationale de protection radiologique);

- Tresques, à environ $10 \mathrm{~km}$ au sud-ouest du site, considéré comme un village non exposé aux rejets du site ;

- Arles, restreinte aux zones inondées du nord de la Camargue en octobre 1993 et en janvier 1994, c'est-à-dire au polygone limité par les lieux-dits Gimeaux, Gageron, Le-Paty-de-la-Trinité, Albaron et Saliers.

Cette étude est divisée en trois chapitres. Le premier chapitre caractérise géographiquement, démographiquement et socio-économiquement les trois communes concernées mais aussi les habitudes alimentaires en ZEATMéditerranée : zone d'étude et d'aménagement du territoire comprenant les deux régions administratives Provence-Alpes-Côte d'Azur et Languedoc et la collectivité territoriale Corse. Le deuxième chapitre est relatif à la méthodologie utilisée: type d'enquête, questionnaire d'enquête, notion «d'unité de consommation ». Le troisième chapitre concerne les résultats. Ceux-ci sont présentés en trois paragraphes : la structure démographique et socio-économique des populations enquêtées, la consommation globale et la part dite d'autoconsommation.

\section{Caractéristiques géographiques, démographiques et socio-économiques des trois communes}

\subsection{Cadre géographique}

Les informations contenues dans ce chapitre sont issues de quatre documents à caractère public : le recensement général de la population réalisé dans le Gard (INSEE, 1990a) et dans les Bouches-du-Rhône en 1990 (INSEE, 1990b), l'enquête concernant les habitudes alimentaires dans la ZEAT-Méditerranée réalisée en 1991 (INSEE, 1991) et l'enquête AGRESTE concernant la population agricole, réalisée en 1988 (MAF, 1988). L'enquête alimentaire réalisée porte sur des habitants sélectionnés de trois communes, en liaison avec le site de Marcoule : Codolet et Tresques dans le département du Gard et Arles dans le département des Bouches-du-Rhône.

La commune de Codolet, dans la pointe constituée par le Rhône et son affluent la Cèze, est située au sud immédiat par rapport au site de Marcoule : le centre du village est en effet à $1,25 \mathrm{~km}$ de la limite sud du site. Elle est globalement considérée comme la plus concernée par les rejets gazeux du site. C'est ce qui a été 


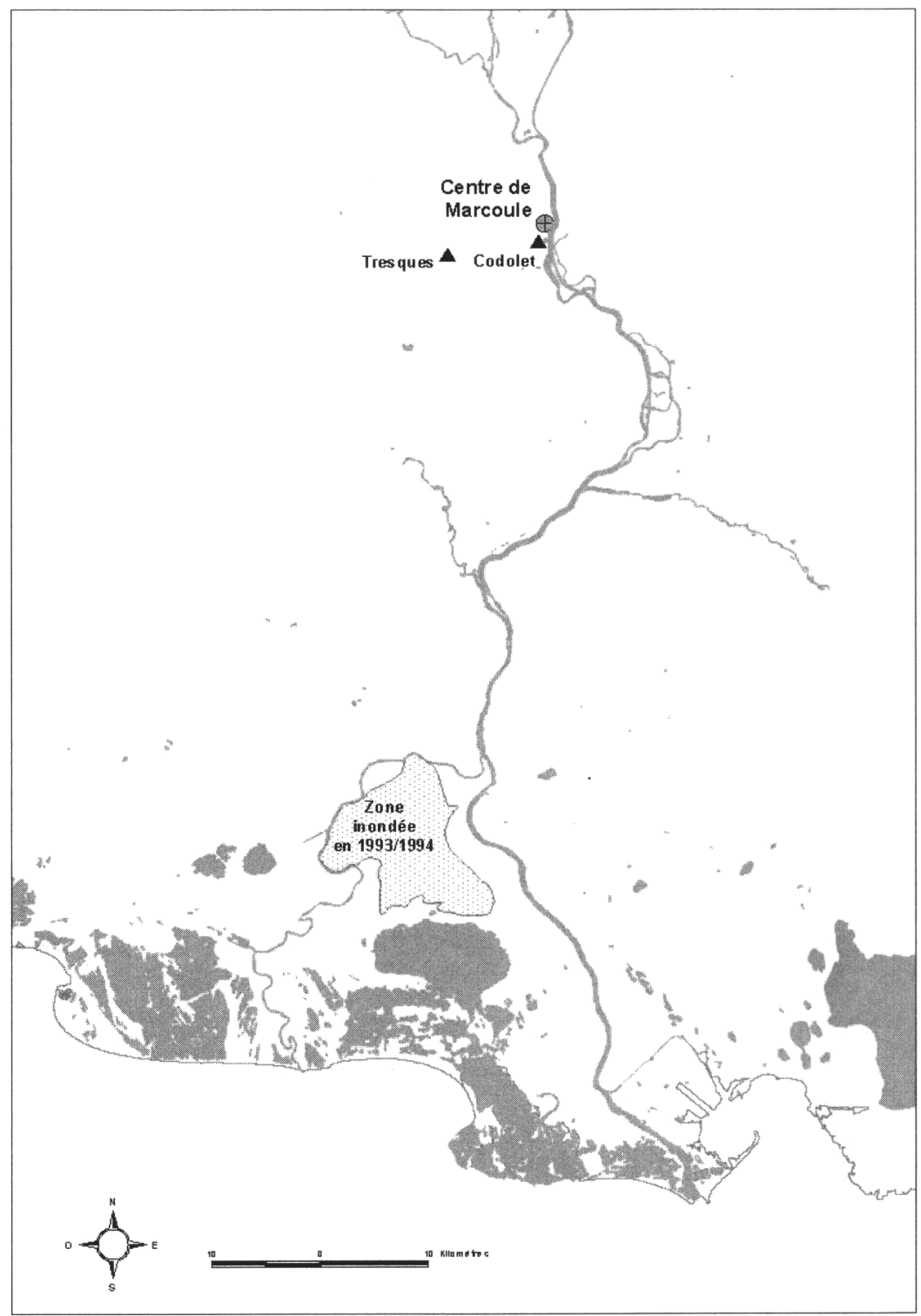

Figure 1 - Positionnement des villages de Codolet et de Tresques et de la zone inondée en 1993 et 1994 par rapport au site de Marcoule.

Location of the villages of Codolet and Tresques and the areas flooded in 1993 and 1994 in relation to the Marcoule site. 
démontré lors d'une étude IPSN relative à l'impact des rejets gazeux tritiés du site sur les feuilles du chêne blanc (Descamps, 1995). Sa superficie est de 517 ha dont 195 réservés aux installations nucléaires et à la Compagnie nationale du Rhône (CNR) et 24 ha en zone habitée.

La commune de Tresques est située dans la vallée de la Tave, à $10 \mathrm{~km}$ au sudouest du site ; elle est considérée, a priori, à l'abri de l'influence des rejets gazeux du site de Marcoule compte tenu des vents dominants de la région (le Mistral de direction principale sud-sud-est) et de l'existence, entre le site et le village, du plateau du Camp de César et du plateau de Lacau. L'étude IPSN précitée a confirmé cet a priori. Sa superficie est de 1790 ha dont 6 ha de centre ancien, 44 ha de quartiers récents, 32 ha en zone d'activité et 415 ha en zones naturelles boisées.

La partie de la commune d'Arles, inondée en octobre 1993 et en janvier 1994, a été retenue dans cette étude puisqu'elle a été l'objet, en ces deux occasions, de dépôts estimés à 400000 tonnes. Ces dépôts sont constitués de sédiments du Rhône qui ont un grand pouvoir de fixation des radionucléides, en particulier du fait de leur fraction argileuse. Les superficies inondées ont atteint les maxima de 12168 ha en octobre 1993 et de 6220 ha en janvier 1994. La zone d'emprise maximale lors les deux périodes d'inondation est de 13700 ha, ce qui représente environ les deux tiers de la Camargue irriguée. Cette zone inondée est située au nord du Vaccarès, dans un quadrilatère délimité par les quartiers (nom local à caractère administratif donné à un hameau ou à un lieu-dit) de Saliers, Albaron et Le-Paty-de-la-Trinité à l'ouest et Gimeaux et Gageron à l'est. À l'intérieur de cette zone les parcelles situées au droit des principales brèches survenues dans la digue (Figarès en 1993 et Lauricet en 1994) ont reçu les dépôts les plus importants.

\subsection{Données démographiques de base}

Ces données sont relatives à 8 classes d'âge : 0-4 ans, 5-9 ans, 10-14 ans, 15-19 ans, 20-39 ans, 40-59 ans, 60-74 ans, $\geq 75$ ans. La comparaison des trois séries de données révèle quelques différences entre les communes.

\section{- Codolet}

Sa population est de 457 habitants dont 452 répartis dans 157 foyers (moyenne de 2,88 habitants par foyer). Les plus de 60 ans (somme des deux dernières classes d'âge) sont $95(20,7 \%$ ) et les moins de 20 ans (somme des quatre premières classes d'âge) sont $124(27,1 \%)$. 


\section{- Tresques}

Sa population est de 1754 habitants dont 1749 répartis dans 607 foyers (moyenne de 2,88 habitants par foyer). Les plus de 60 ans sont $274(15,6 \%)$ et les moins de 20 ans sont $485(27,6 \%)$.

\section{- Arles}

Sa population est de 52063 habitants dont 50928 répartis dans 19740 foyers (moyenne de 2,58 habitants par foyer). Les plus de 60 ans sont $11518(22,1 \%)$ et les moins de 20 ans sont 13268 (25,5\%). La mairie d'Arles estime à 500 le nombre de foyers susceptibles d'avoir été concernés par les inondations ; sur la base d'une moyenne de 2,58 habitants par foyer, cette estimation correspond à une population de l'ordre de 1300 personnes.

\subsection{Données démographiques à caractère agricole et données sur l'agriculture}

\section{- Codolet}

Sa population agricole (somme de la population agricole familiale et des salariés agricoles permanents) est de 103 personnes, soit $22,5 \%$ de la population. Sur ces 103 personnes, 50 sont issues des familles des exploitants «actives sur l'exploitation », parmi lesquelles il y a 17 chefs d'exploitation à temps complet ou à mi-temps. La superficie agricole utile (S.A.U.) est de 274 ha et la principale culture est la vigne avec 233 ha. Les vergers avec 18 ha et une petite production de légumes frais avec 2 ha sont aussi à signaler. La surface irrigable est de 37 ha. La superficie cumulée de jachères et de jardins familiaux est de 16 ha.

\section{- Tresques}

Sa population agricole totale est de 273 personnes, soit $15,6 \%$ de la population. Sur ces 273 personnes, 177 sont issues des familles d'exploitants « actives sur l'exploitation », parmi lesquelles il y a 59 chefs d'exploitation à temps complet ou à mi-temps. La S.A.U. est de 1120 ha et la principale culture est la vigne avec 786 ha. Les légumes frais avec 97 ha, les vergers avec 71 ha et les céréales avec 34 ha sont aussi à signaler. La surface irrigable est de 39 ha. La superficie cumulée des jachères et jardins familiaux est de 73 ha.

\section{- Arles}

Sa population agricole totale est de 2608 personnes, soit $5 \%$ de la population. Sur ces 2608 personnes, 1086 sont issues des familles d'exploitants « actives sur l'exploitation ", parmi lesquelles il y a 443 chefs d'exploitation à temps complet ou à mi-temps. 
En Camargue, 15000 à 20000 ha sont irrigués sur les 26000 irrigables. Les principales cultures sont les céréales (riz, blé) avec 18089 ha, les cultures industrielles avec 2815 ha, le fourrage en culture principale avec 1677 ha, les légumes frais et en particulier les pommes de terre avec 794 ha, les vergers avec 769 ha ; les surfaces toujours en herbe (S.T.H.) étant de 19145 ha. La superficie cumulée de jachères et de jardins familiaux est de 989 ha.

\section{Caractéristiques de l'autoconsommation}

Les activités favorisant l'autoconsommation sont de trois types: le jardinage, l'élevage et l'ensemble chasse et pêche.

- Le jardinage

Les jardins de Codolet, village très concentré, sont généralement groupés autour des maisons ; les jardins les plus importants étant situés sur la périphérie du village où ils occupent notamment l'espace situé de part et d'autre de la digue entre le bras du Rhône, la lône et les maisons du village. On retrouve par ailleurs des jardins plus ou moins englobés dans les cours de ferme, vers l'ouest. Tous ces jardins sont irrigués par la nappe phréatique à $10 \mathrm{~m}$. Quelques rares propriétaires arrosent cependant leur jardin avec l'eau du forage communal, profond.

Les jardins de Tresques sont de trois types compte tenu de leur localisation : des petits jardins traditionnels situés dans la vallée, entre la Tave et le village, des jardins relativement importants autour d'un habitat rural assez dispersé et des «jardins » avec de très petites parcelles de maraîchage dans les lotissements récents. Les ressources en eau pour l'arrosage sont abondantes. Elles sont alimentées par la vallée de la Tave et la cuvette de l'étang et elles sont soutenues par les abondantes ressources souterraines, accumulées dans le plateau de Sabran au nord-ouest.

En Camargue, le jardin potager ou le verger, passe souvent inaperçu dans ces espaces immenses mais il est généralement présent dans des conditions sociologiques analogues aux deux autres zones. Les jardins de la Camargue arlésienne sont développés autour des hameaux et des îlots d'habitats souvent attenants aux exploitations, les mas. On notera que beaucoup des jardins de Camargue souffrent des inondations, mais aussi de la gestion des rizières et des mauvais drainages des étiers et canaux vers l'étang de Vaccarès.

\section{- L'élevage}

Dans les trois zones, les élevages se limitent essentiellement à des petits élevages individuels de basse-cour, essentiellement de poulet. À Tresques, on recense aussi 
un élevage de caprins qui donne lieu à une autoconsommation, faible et irrégulière, de fromage dans le village. En Camargue l'élevage n'est pas, contrairement à ce qu'on pourrait croire, une activité intensive. Elle est cependant une terre d'élevage traditionnel des taureaux par l'intermédiaire des manades. Cet élevage a été récemment re-dynamisé par la création d'une appellation A.O.C. À ceci s'ajoutent quelques élevages artisanaux de volailles dont celui de Gimeaux qui est le plus important.

- La chasse et la pêche

À Codolet, où le territoire communal est assez restreint, une grande partie des activités de chasse est pratiquée hors de la commune. La pêche est pratiquée dans le lac, ainsi d'ailleurs que par les habitants des villages avoisinants. À Tresques, la chasse est pratiquée, notamment dans les bois qui entourent le village. En Camargue on pratique la chasse aux oiseaux et la pêche, aussi bien en eau douce (étangs, deux bras du Rhône, canaux de drainage) qu'en eau de mer (en estuaire du Petit-Rhône essentiellement).

\section{Méthode d'étude pour l'enquête alimentaire}

\subsection{Détermination du type d'enquête}

Pour ce qui concerne la connaissance de l'alimentation des populations on peut avoir recours à deux catégories d'étude : le bilan des disponibilités alimentaires d'une part, les enquêtes alimentaires d'autre part.

Le bilan des disponibilités alimentaires est utilisable à condition de connaître certaines données statistiques relatives au nombre d'individus concernés, à la production alimentaire, aux échanges commerciaux, aux stocks, etc. Globalement on peut dire que cette méthode est applicable à un pays et, à la rigueur, à une région, mais pas à un groupe restreint, comme un village. De plus, cette méthode ne peut pas donner d'indications sur les différentes couches de population.

Les enquêtes alimentaires, réalisées par des organismes ou des sociétés différents, avec des buts différents et selon des méthodes différentes ne sont en général pas comparables. La principale difficulté est donc de définir la méthode appropriée au but recherché. Il y a 4 catégories d'enquête : celle relative à un pays ou à une ville, celle relative à un groupe homogène de la population, celle de type «familiale » et celle de type «individuelle». Dans le cas présent, ce sont les deux dernières catégories qui peuvent être envisagées. L'enquête individuelle est en théorie la plus intéressante mais elle se heurte à la difficulté de mise en application du fait de la quantité de personnel nécessaire. C'est donc la méthode 
«familiale » qui présente le meilleur compromis. Elle présente l'inconvénient de devoir nécessiter une conversion pour passer d'une consommation familiale à une consommation individuelle moyenne. On estime en général qu'une vingtaine de familles par zone est suffisante pour des populations de l'ordre du millier (environ 500 à Codolet, 2000 à Tresques et 1300 en zone camarguaise inondée).

Le choix de « l'échantillon à étudier » dans chacune des trois enquêtes avait la prétention d'être représentatif de chacune des trois populations concernées. Parmi les méthodes de tirage des enquêtés, le tirage aléatoire est théoriquement le plus approprié mais il ne permet pas de tenir compte de manière simple de l'hétérogénéité de la population. La méthode d'échantillonnage retenue est donc la méthode dite des « quotas », communément appliquée par les instituts de sondage. Elle permet notamment de mieux contrôler que les autres méthodes de tirage l'hétérogénéité et la structure de la population et elle fournit de ce fait des échantillons plus fidèles. Trois critères principaux ont été retenus pour diriger le «choix » des échantillons à enquêter :

- le coût de l'enquête puisque celui-ci conditionne le nombre de questionnaires d'enquête déposé ;

- la meilleure représentation des foyers des deux villages et de la zone camarguaise inondée en tenant compte de l'hétérogénéité spatiale des communes et de l'existence de différentes « composantes ». Nous en citerons trois : les enfants en bas âge dont on sait qu'ils ont un régime alimentaire particulier, les diverses catégories socio-économiques à comportements alimentaires susceptibles d'être différents (agriculteurs, artisans, commerçants, chefs d'entreprise, cadres, employés et ouvriers, inactifs) et surtout les possesseurs de jardins, de vergers et de petits élevages, les chasseurs et les pêcheurs qui ont traditionnellement des habitudes d'autoconsommation importantes, pour eux-mêmes et leurs familles mais aussi, indirectement, pour des « amis »;

- l'acceptation du questionnaire d'enquête par les foyers consultés et la perception par l'enquêteur d'une grande probabilité de retour de ce questionnaire. Notons qu'à cet égard l'attitude des élus contactés a eu une influence inégale.

In fine, nous avons retenu :

- des foyers ${ }^{3}$, avec ou sans enfants, de tranches d'âge différentes mais qui pour l'exploitation des données seront répartis en seulement trois catégories d'âge : plus de 12 ans, entre 12 et 4 ans, et moins de 4 ans (les célibataires sont exclus de l'enquête) ;

\footnotetext{
${ }^{3}$ Le foyer est une cellule familiale : un célibataire ne peut donc pas constituer un foyer.
} 
- des foyers dont le chef de famille appartient à des milieux socio-économiques et culturels différents ;

- des foyers où les sources potentielles d'autoconsommation sont réelles : possesseurs de jardins et de vergers, chasseurs, pêcheurs (ou en relation de famille ou d'amitié avec eux).

\section{2. Élaboration du questionnaire}

Le questionnaire se présente sous la forme d'un cahier d'enquête de 14 pages, dit par échelle. Ce questionnaire est standardisé, ordonné par thème, avec questions qualitatives et/ou quantitatives. Il a deux objectifs majeurs :

- connaître les usages et les consommations alimentaires des foyers ;

- connaître les pratiques d'autoconsommation des foyers et la proportion de l'autoconsommation intra-communale ou locale dans la consommation des foyers.

La constitution précise du cahier d'enquête est la suivante :

- page 1 : localisation et présentation du foyer (aspect sociologique et socioéconomique) ;

- page 2 : compositions de la population familiale concernée par chacun des trois repas principaux lors des 7 jours de la même semaine ;

- pages 3 à 9 : descriptions des menus pour chacun des trois repas principaux lors des 7 jours de la même semaine (y compris les produits d'autoconsommation) ;

- page 10 : estimations de la consommation hebdomadaire des produits de base (pain, lait, œufs, sucre, corps gras, boissons, etc.) et habitudes des petits repas (goûter, grignotage) ;

- pages 11 à 14 : renseignements sur l'autoconsommation à partir de l'exploitation du jardin et de l'élevage familial, de la chasse, de la pêche et de la cueillette. Ces renseignements permettent une estimation des quantités annuelles autoconsommées.

\subsection{Denrées susceptibles d'être retenues}

Des listes pré-établies de denrées ont été proposées pour les questionnaires d'enquête relatifs aux «produits de base» et à «l'autoconsommation d'exploitation, de prédation et de cueillette ». Ces listes ne devaient toutefois pas être considérées comme rigides afin de permettre l'expression des particularités.

- Liste des produits de base

Cette liste comprend le pain, les æufs, le lait, le sel, le sucre, les corps gras (beurre, margarine, huiles) et les boissons (eaux diverses, vin, cidre, boissons gazeuses 
sucrées, jus de fruit). Pour les œufs, le sel, l'huile d'olive, le vin, l'eau de robinet et l'eau de forage, la provenance de ces produits était demandée.

- Liste des produits susceptibles d'être autoconsommés

Cette liste comprend des produits de potager (légumes), de verger (fruits), de cueillette (champignons, figues, pissenlit, asperge sauvage, thym, romarin...), d'élevage (poulet, lapin...), de chasse, de pêche, de ramassage (escargots, coquillages divers...). Pour l'ensemble de cette liste, il y avait lieu aussi de préciser la provenance de ces produits. Compte tenu du caractère saisonnier de l'enquête on peut penser que les données relatives aux produits autoconsommés de saison sont un peu majorés et que les autres (par exemple pomme de terre et pomme) sont un peu minorés.

\subsection{Caractéristiques générales de la mise en place de l'enquête}

D'une manière générale, l'enquête est conduite sous la forme d'entretiens semidirectifs dont on a défini précédemment les objectifs.

Un entretien liminaire de 10 à 30 minutes entre l'enquêteur et son « correspondant » est nécessaire pour apprécier si le foyer répond aux besoins de l'échantillonnage, pour présenter le questionnaire, pour obtenir l'agrément du foyer, et dans ce cas, pour donner les consignes adéquates et traiter à titre d'exemple quelques questions. Le retour ${ }^{4}$ de l'enquêteur auprès du même « correspondant » s'effectue sur rendez-vous. La relecture du cahier d'enquête a lieu durant un nouvel entretien, d'une demi-heure à une heure, selon la qualité des différentes informations recueillies, en particulier celles sur les pratiques d'autoconsommation.

\subsection{Unité de consommation ou « uc»}

Il a été dit précédemment que l'enquête hebdomadaire nécessitait de devoir recourir à la transformation d'une consommation familiale et hebdomadaire, la donnée de base recueillie, en une consommation individuelle et journalière, ceci afin de permettre des comparaisons entre différentes études. Cette nécessité conduit à définir l' «unité de consommation» ou «uc ». Cette uc est liée au nombre et à la qualité des personnes présentes à chacun des trois repas principaux (petit déjeuner, déjeuner et dîner) et pour chacun des sept jours de la même semaine (Cresta et al., 1969). Pour effectuer ce calcul, il est donc nécessaire de

\footnotetext{
4 Un délai maximum de deux à trois semaines est laissé à chaque « correspondant " pour apporter sa contribution à l'enquête ct ceci dans le but de garantil' une période d'enquête commune à tous les foycrs.
} 
connaître la somme totale des repas servis pendant toute la semaine au sein du foyer.

La détermination de l'unité de consommation est différente selon les études. Pour notre enquête nous avons choisi de retenir le principe des «coefficients" comme dans l'enquête alimentaire de l'INSEE de $1991^{5}$. Dans notre enquête les enfants de 1,2 et 3 ans n'ont pas été retenus compte tenu de leur grande spécificité en matière d'alimentation. Pour les enfants, entre 4 et 12 ans inclus, nous avons affecté un coefficient de 0,5 et pour les personnes à partir de 13 ans nous avons retenu un coefficient de 1 . Pour un aliment donné on passe donc d'une consommation hebdomadaire du foyer en gramme ou en litre $\left(\mathbf{Q}_{\mathrm{h}, \mathrm{f}}\right)$ à une consommation journalière par unité de consommation en gramme ou en litre $\left(\mathbf{Q}_{\mathrm{j}, \mathrm{uc}}\right)$ par la formule :

$$
\mathbf{Q}_{\mathrm{j}, \mathrm{uc}}=\mathbf{Q}_{\mathrm{h}, \mathrm{f}} /(\text { nombre uc } * 7) \text {. }
$$

Si, comme le précise l'INSEE, la notion d'uc n'est qu'un concept, elle se révèle néanmoins très utilisée et reconnue satisfaisante pour la pondération qu'elle apporte à la consommation «brute » du foyer par rapport au nombre et à la qualité des membres de ce foyer pendant la période d'enquête. Elle tient donc compte d'une certaine "réalité » de la consommation au sein du foyer. Dans la réalité, l'expression des résultats en « gramme ou en litre par unité de consommation et par jour » est assez peu différente de celle, plus usuelle, en « gramme ou en litre par habitant et par jour ».

\subsection{Dépouillement de l'enquête}

\section{- Attitude des municipalités}

Afin de faciliter le déroulement de l'enquête, l'IPSN a organisé une information préalable auprès des maires de Codolet, Tresques et Arles par l'intermédiaire d'un courrier en date du 9 janvier 1998. Cette information a été étendue au président de la Commission locale d'information du Gard, aux présidents des Conseils régionaux de Provence-Alpes-Côte d'Azur et de Languedoc-Roussillon, aux préfets, présidents des Conseils généraux et directeurs départementaux de l'agriculture et de la forêt du Gard, du Vaucluse et des Bouches-du-Rhône. De plus, une réunion d'information publique a été organisée par la mairie d'Arles, à Saliers le 9 avril 1998, en présence des habitants de la zone enquêtée en Camargue et en présence de journalistes locaux.

\footnotetext{
5 Dans cette enquête le chef de famille compte pour I, les autres membres du foyer de plus de 13 ans comptent pour 0,7 et les moins de 13 ans pour 0,5 . Le coefficient de 1 pour le seul chef de famille et le coefficient de 0,5 pour tous les enfants de moins de 13 ans (y compris ceux de moins de 3 ans) nous paraissent être un mauvais choix.
} 
- Répartition des questionnaires

Au total 83 questionnaires ont été distribués après avoir contacté une centaine de foyers et 80 d'entre eux ont été récupérés. Le nombre total de questionnaires validés et conservés est de 65 : 25 en Camargue, 22 à Codolet et 18 à Tresques; ce qui représente environ 200 personnes. Le dépouillement des questionnaires totalise 333 variables numériques (var.) : description des familles (37 var.), alimentation de base (32 var.), enquête hebdomadaire (116 var.), autoconsommation (90 var.), production de jardin (58 var.).

\section{Résultats}

\subsection{Consommation}

\subsubsection{Consommation globale}

- Aliments solides

La consommation totale en aliments solides est relativement proche pour les trois sites : $1555 \mathrm{~g} \mathrm{uc}^{-1} \mathrm{j}^{-1}$ à Codolet, 1452 en Camargue et 1445 à Tresques et la moyenne totale générale est de $1485 \mathrm{~g} \mathrm{uc}^{-1} \mathrm{j}^{-1}$.

Les consommations pour les 9 catégories d'aliments solides sont présentées sur la Figure 2. En fonction de la moyenne pour les trois échantillons, on obtient le classement décroissant suivant (en $\mathrm{g} \mathrm{uc}^{-1} \mathrm{j}^{-1}$ ): les légumes-fruits (240), les viandes (225), les céréales et assimilés (220), les légumes-racines (214), les fruits (175), les légumes-feuilles (142), les produits laitiers (135), l'ensemble poissons, crustacés et coquillages (58) et les œufs (35). Pour chacune des 9 catégories, les variations sont assez faibles entre les trois échantillons enquêtés. Quelques commentaires peuvent être apportés.

Chacune des trois catégories de légumes est dominée par une espèce : la salade pour les légumes-feuilles (moyenne de $80 \mathrm{~g} \mathrm{uc}^{-1} \mathrm{j}^{-1}$ ), la pomme de terre pour les légumes-racines (moyenne de $130 \mathrm{~g} \mathrm{uc}^{-1} \mathrm{j}^{-1}$ ) et la tomate pour les légumes-fruits (moyenne de $66 \mathrm{~g} \mathrm{uc}^{-1} \mathrm{j}^{-1}$ ). Pour les fruits l'ensemble pomme/poire, largement dominant, présente des variations importantes : $113 \mathrm{~g} \mathrm{uc}^{-1} \mathrm{j}^{-1}$ à Codolet, $55 \mathrm{~g} \mathrm{uc}^{-1} \mathrm{j}^{-1}$ à Tresques et $42 \mathrm{~g} \mathrm{uc}^{-1} \mathrm{j}^{-1}$ en Camargue. Les viandes sont surtout constituées de viande d'élevage ; le bœuf, le porc et les volailles (surtout le poulet) représentent chacun environ $65 \mathrm{~g} \mathrm{uc}^{-1} \mathrm{j}^{-1}$. Les céréales et assimilés sont constitués essentiellement du pain (environ $70 \%$ ), du riz, des pâtes et des pâtisseries. Les produits laitiers concernent les yaourts $(60 \%)$ et les fromages. 


\section{B. DESCAMPS, F. GUILLET}

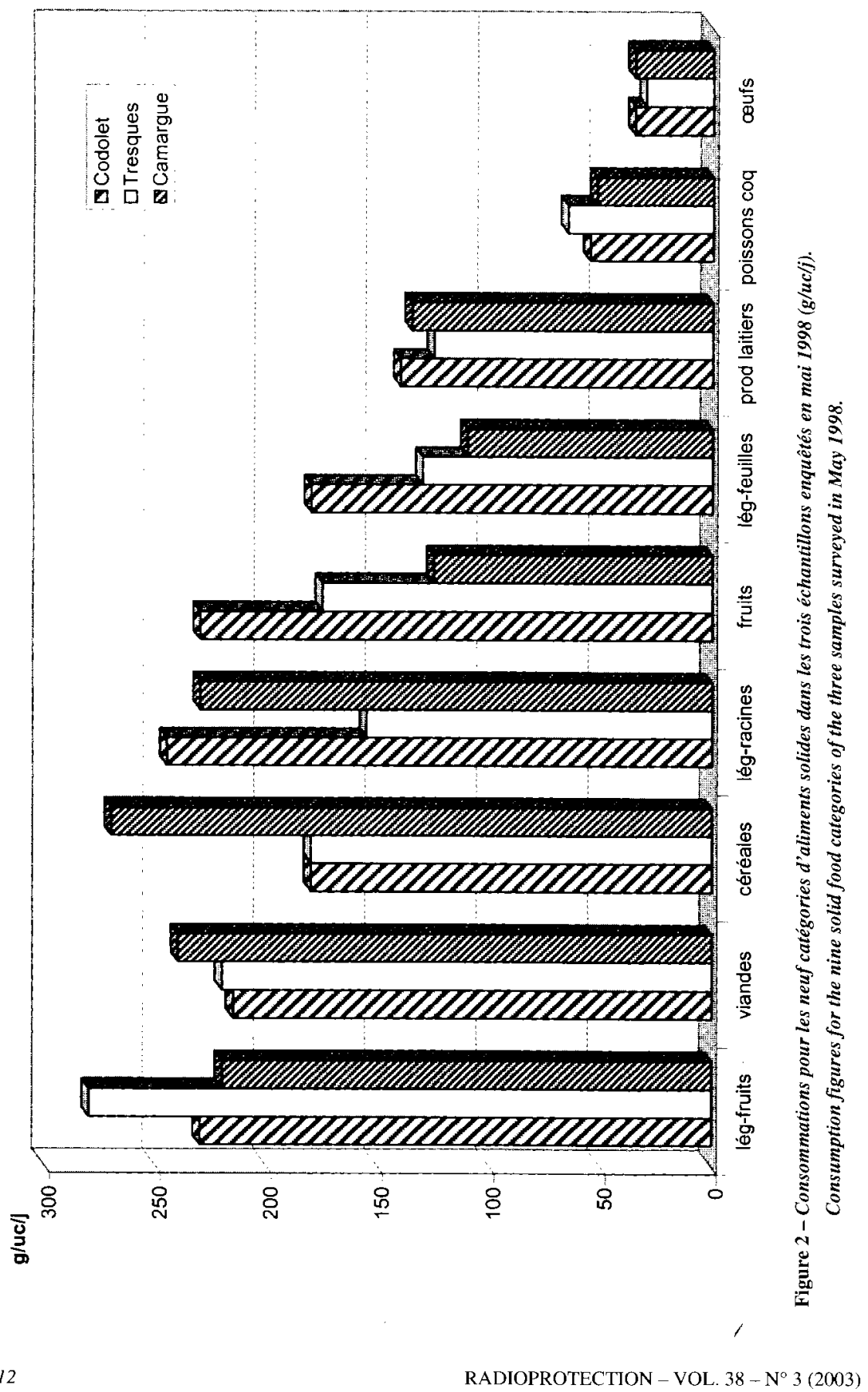


- Aliments liquides

Ils concernent les boissons stricto sensu comme les eaux, le lait, le vin, les autres boissons alcoolisées (bière, cidre), les boissons gazeuses et/ou sucrées (soda, sirop, jus), le thé et le café mais aussi les autres liquides alimentaires comme les soupes de légumes et les potages instantanés. La consommation totale moyenne des aliments liquides est de $21 \mathrm{uc}^{-1} \mathrm{j}^{-1}$ dont 1,75 pour les seules boissons. Cette consommation totale est un peu plus importante en Camargue $\left(2,361 \mathrm{uc}^{-1} \mathrm{j}^{-1}\right)$ que dans les deux autres zones $\left(21 \mathrm{uc}^{-1} \mathrm{j}^{-1}\right.$ à Codolet et $1,731 \mathrm{uc}^{-1} \mathrm{j}^{-1}$ à Tresques). La Figure 3 présente les principaux résultats pour ces aliments liquides. Quelques commentaires peuvent être apportés.

L'eau consommée dans la zone d'étude a trois types de provenance. L'eau de robinet, associée en général à des forages profonds, et de ce fait non considérée comme une eau «locale», est la plus consommée : Codolet $\left(0,4 \mathrm{luc}^{-1} \mathrm{j}^{-1}\right)$, Tresques $\left(0,651 \mathrm{uc}^{-1} \mathrm{j}^{-1}\right)$, Camargue $\left(0,8 \mathrm{luc}^{-1} \mathrm{j}^{-1}\right)$. Les eaux minérales du commerce sont consommées, pour leur qualité gustative, en particulier en Camargue $\left(0,61 \mathrm{uc}^{-1} \mathrm{j}^{-1}\right)$ en raison des mauvaises qualités des autres eaux. L'eau de source est essentiellement consommée à Tresques $\left(0,2 \mathrm{l} \mathrm{uc}^{-1} \mathrm{j}^{-1}\right)$, plus précisément à la « Source des Lions", au centre du village. Le lait est consommé à peu près en égale quantité dans les trois zones, la moyenne étant de $0,241 \mathrm{uc}^{-1} \mathrm{j}^{-1}$. C'est aussi le cas du vin consommé à une moyenne de $0,14 \mathrm{luc}^{-1} \mathrm{j}^{-1}$. Il est quasi exclusivement issu de la cave-coopérative locale à Tresques et à Codolet.

\subsubsection{Classification des aliments en fonction de la quantité et de la variabilité de la consommation}

Cette classification est réalisée en fonction de trois paramètres : la moyenne, le coefficient de variation (C.V) et la médiane. La moyenne détermine si un aliment est fortement ou faiblement consommé. On a retenu pour les aliments fortement consommés la limite de $100 \mathrm{~g} \mathrm{uc}^{-1} \mathrm{j}^{-1}$ pour les aliments solides et de $1 \mathrm{l} \mathrm{uc}^{-1} \mathrm{j}^{-1}$ pour les aliments liquides. Les deux autres paramètres déterminent si la consommation est peu variable ou très variable. Le coefficient de variation, rapport entre l'écart-type et la moyenne, est une valeur sans unité. Quand il est inférieur à 1 , on considère que la consommation est peu variable ; il peut atteindre 4 ou 5 pour des consommations très variables. Si la médiane est égale à 0 , c'est-à-dire si au moins $50 \%$ des effectifs ne consomment pas le produit, on considère aussi que la consommation est très variable puisque peu fréquente. Compte tenu de ces trois paramètres, les aliments appartiennent à trois catégories :

- aliments de consommation forte et peu variable : moyenne $>100 \mathrm{~g} \mathrm{uc}^{-1} \mathrm{j}^{-1}$ ou $>1 \mathrm{luc}^{-1} \mathrm{j}^{-1}$, coefficient de variation $<1$, médiane non nulle. Le tableau I 


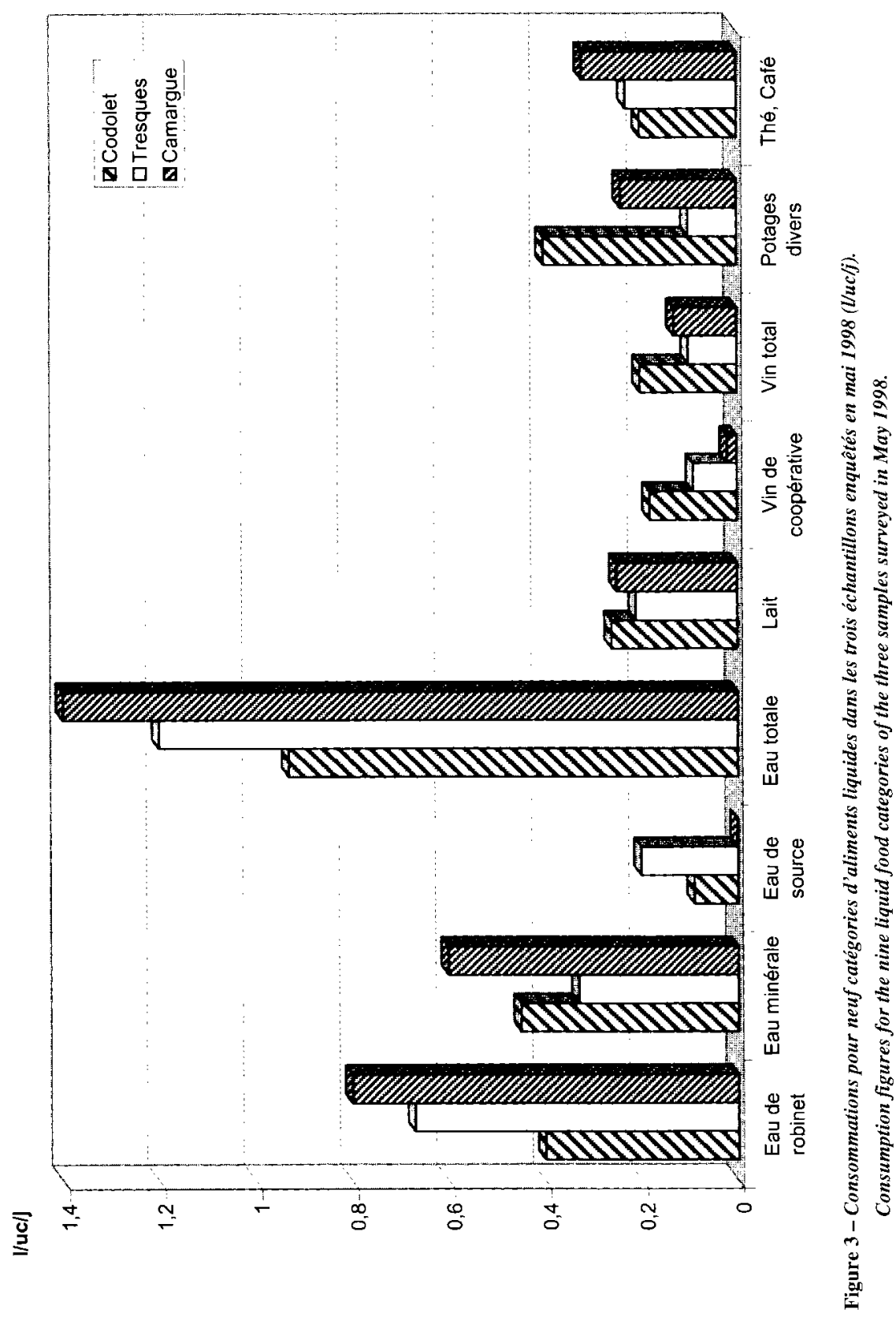


présente, pour les trois échantillons enquêtés, la moyenne et le coefficient de variation de 5 groupes d'aliments et des 2 seuls aliments (pomme de terre et pain) entrant dans cette catégorie. Ces aliments sont généralement consommés par tous les foyers ;

\section{TABLEAU I}

Aliments de consommation forte et peu variable (moyenne en $\mathrm{g} \mathrm{uc}^{-1} \mathrm{j}^{-1}$ et coefficient de variation sans unité).

High consumption, low variable consumption foodstuffs (mean values in $\mathrm{g} \mathrm{uc}^{-1} \mathrm{j}^{-1}$ and coefficient of variation without unit).

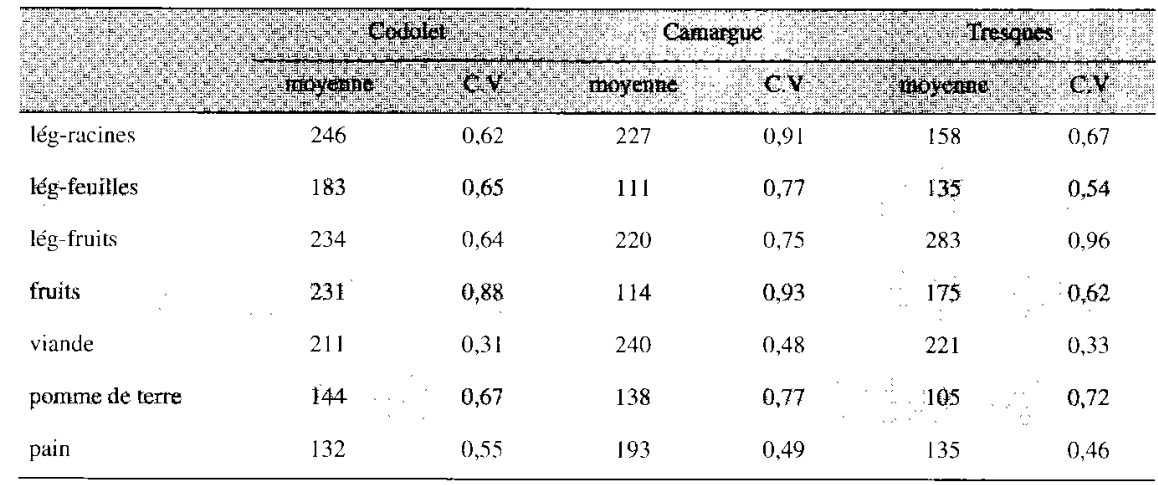

- aliments de consommation faible et peu variable : moyenne $<100 \mathrm{~g} \mathrm{uc}^{-1} \mathrm{j}^{-1}$ ou $<1 \quad u_{u c}^{-1} j^{-1}$, coefficient de variation $<1$, médiane non nulle. Dans cette catégorie figurent les aliments consommés par la totalité ou par une grande proportion des foyers de chacune des trois échantillons enquêtés mais en faible ou assez faible quantités. On a également mis dans ce groupe les aliments pour lesquels le coefficient de variation est légèrement supérieur à 1 , dans une seule des trois zones. Le tableau II est relatif aux 14 aliments de cette catégorie ;

- aliments de consommation faible et très variable : moyenne $<50 \mathrm{~g} \mathrm{uc}^{-1} \mathrm{j}^{-1} \mathrm{ou}$ $<0,51 \mathrm{uc}^{-1} \mathrm{j}^{-1}$, coefficient de variation $>1$, médiane nulle. 53 aliments sont concernés : 23 légumes et 10 fruits, 9 produits d'origine animale, 5 produits sucrés et 6 produits liquides. Parmi ces 53 aliments on peut retenir les aliments consommés, dans chacune des trois zones, en quantité supérieure à $10 \mathrm{~g} \mathrm{uc}^{-1} \mathrm{j}^{-1}$ pour les solides et à $0,31 \mathrm{uc}^{-1} \mathrm{j}^{-1}$ pour les liquides. Il s'agit des asperges, des carottes, des courgettes, des haricots verts, des petits pois, des poireaux et des oignons pour les légumes, des cerises, des fraises, des pêches et des pommes/poires pour les fruits, de la charcuterie, de la confiserie, de la pâtisserie et des poissons de mer pour les autres aliments solides, de l'eau minérale et de l'eau de robinet pour les liquides. 


\section{TABLEAU II}

Aliments de consommation faible et peu variable (moyenne en $g \mathbf{u c}^{-1} \mathrm{j}^{-1}$ ou en I uc $\mathrm{uc}^{-1} \mathrm{j}^{-1}$ et coefficient de variation sans unité).

Low consumption, low variable consumption foodstuffs (mean values in $\mathrm{uc}^{-1} \mathrm{j}^{-1}$ and coefficient of variation without unit).

\begin{tabular}{|c|c|c|c|c|c|c|}
\hline & Tod & & 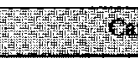 & & Py & 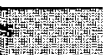 \\
\hline 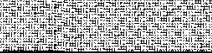 & norne & $\mathrm{CN}$ & Notone & $\mathrm{CV}$ & mosy & Cyt \\
\hline salade & 101 & 0,55 & 61 & 0,63 & 78 & 0,52 \\
\hline fonate & 62 & 0,93 & 56 & 1,51 & 84 & 0,88 \\
\hline porc & 71 & 0,48 & 54 & 0,77 & 74 & 0,69 \\
\hline bouf & 70 & 0,57 & 77 & 0,581 & 70 & 0,72 \\
\hline poulet & 36 & 0,95 & 42 & 1,16 & 39 & 0,87 \\
\hline lait & 027 & 0,93 & 026 & 0,72 & 0,21 & 1,36 \\
\hline fromage & 60 & 0,62 & 55 & 0,64 & 61 & 0,59 \\
\hline yaourt & 82 & 0,9 & 81 & 1,06 & 64 & 0,93 \\
\hline cufs & 36 & 0,71 & 36 & 0,7 & 30 & 0,85 \\
\hline poisson/coquillage & 53 & 0,57 & 57 & 1,6 & 64 & 0,89 \\
\hline pâtes & 25 & 0,84 & 31 & 1 & 19 & 0,68 \\
\hline iz & 8 & 136 & 18 & 0,93 & 14 & 086 \\
\hline sucre & 14 & 0,95 & 20 & 0,76 & 13 & 0,85 \\
\hline ean de robinet. & 0,39 & 1,3 & 0,79 & 0,85 & 0.67 & 076 \\
\hline
\end{tabular}

\subsection{Autoconsommation}

\subsubsection{Quantités autoconsommées pour les principaux aliments}

Sur les 39 fruits et légumes recensés dans l'enquête, 32 sont autoconsommés, dans au moins une zone. On en retient 9 autoconsommés de façon significative, c'est-àdire avec des moyennes pour les trois zones supérieures à $10 \mathrm{~g} \mathrm{uc}^{-1} \mathrm{j}^{-1}$. Il s'agit par ordre décroissant : la pomme de terre $(62 \mathrm{~g})$, la salade $(42 \mathrm{~g})$, la tomate $(18 \mathrm{~g})$, la carotte (16 g), le poireau, le haricot vert et la fraise $(13 \mathrm{~g})$, la cerise et le radis $(11 \mathrm{~g})$. La carotte, la pomme de terre et le poireau sont plus autoconsommés en Camargue, la tomate et le haricot vert à Tresques, la fraise et la cerise à Codolet. Sept autres légumes sont autoconsommés dans les trois zones mais avec des moyennes pour les trois zones inférieures à $10 \mathrm{~g} \mathrm{uc}^{-1} \mathrm{j}^{-1}$. Il s'agit par ordre décroissant : le melon $(8 \mathrm{~g})$, le petit pois $(7 \mathrm{~g})$, la courgette $(6 \mathrm{~g})$, la blette et le céleri $(4 \mathrm{~g})$, le navet $(3 \mathrm{~g})$ et le haricot $\sec (2 \mathrm{~g})$. En terme de fréquence d'autoconsommation on notera qu'elle est importante (environ $60 \%$ des foyers) pour la pomme de terre et la salade en Camargue et pour la salade à Codolet. 
Elle est par contre faible (moins de $30 \%$ des foyers) pour la tomate en Camargue, les trois légumes à Tresques et la tomate à Codolet.

Sur les 17 produits d'origine animale seuls les œufs et le poulet sont autoconsommés dans les trois zones et ceci avec des moyennes de $15 \mathrm{~g} \mathrm{uc}^{-1} \mathrm{j}^{-1}$. Pour ces 2 aliments, l'autoconsommation à Codolet est significativement plus faible. Par ailleurs le fromage $\left(7 \mathrm{~g} \mathrm{uc}^{-1} \mathrm{j}^{-1}\right)$ et les poissons d'eau douce $\left(16 \mathrm{~g} \mathrm{uc}^{-1} \mathrm{j}^{-1}\right)$ à Tresques, les poissons de mer $\left(5 \mathrm{~g} \mathrm{uc}^{-1} \mathrm{j}^{-1}\right)$ et le bœuf/taureau $\left(7 \mathrm{~g} \mathrm{uc}^{-1} \mathrm{j}^{-1}\right.$ ) en Camargue sont à retenir. Seuls les cufs en Camargue présentent une fréquence d'autoconsommation importante $(65 \%)$. La fréquence est faible (de l'ordre de $20 \%$ des foyers) pour les œufs à Tresques et à Codolet et le poulet en Camargue. Elle très faible ou nulle (moins de $10 \%$ des foyers) pour les poulets à Tresques et à Codolet et le fromage/yaourt dans les trois zones.

Pour les liquides seuls le vin et l'eau sont concernés par une autoconsommation moyenne supérieure à $10 \mathrm{cl} \mathrm{uc}^{-1} \mathrm{j}^{-1}$; le lait n'est pas du tout autoconsommé. L'autoconsommation de vin (vin de la coopérative locale) est deux fois plus importante à Codolet qu'à Tresques. En terme de fréquence, presque tous les foyers de Codolet et de Tresques sont concernés contre seulement $10 \%$ des foyers en Camargue.

Pour l'eau dite « locale », on notera qu'elle est absente en Camargue (très mauvaises qualités organoleptiques), qu'elle concerne essentiellement la source des Lions à Tresques pour presque tous les foyers et qu'elle n'est consommée que par un foyer (mais en grande quantité) à Codolet.

La figure 4 récapitule les résultats pour les 13 aliments solides et liquides dont l'autoconsommation moyenne pour les trois zones est supérieure à $10 \mathrm{~g} \mathrm{uc}^{-1} \mathrm{j}^{-1}$ ou à $10 \mathrm{cl} \mathrm{uc}^{-1} \mathrm{j}^{-1}$.

\subsubsection{Incidence de l'autoconsommation constatée en 1998 pour le calcul de l'impact dosimétrique lié à l'ingestion sur la population du village de Codolet}

L'enquête alimentaire réalisée au printemps 1998 avait essentiellement pour but de déterminer, avec le plus de précision possible, la part de la consommation alimentaire des habitants de Codolet susceptible d'avoir été influencée par les rejets atmosphériques et liquides du site de Marcoule. Les autoconsommations relatives à Codolet de la figure 4 représentent la part maximale de la consommation des habitants du village susceptible d'avoir été contaminée par le site. Cette autoconsommation « réelle et actualisée » peut être comparée à celle utilisée, pour l'année 1993, dans le calcul d'impact dosimétrique du site pour les 


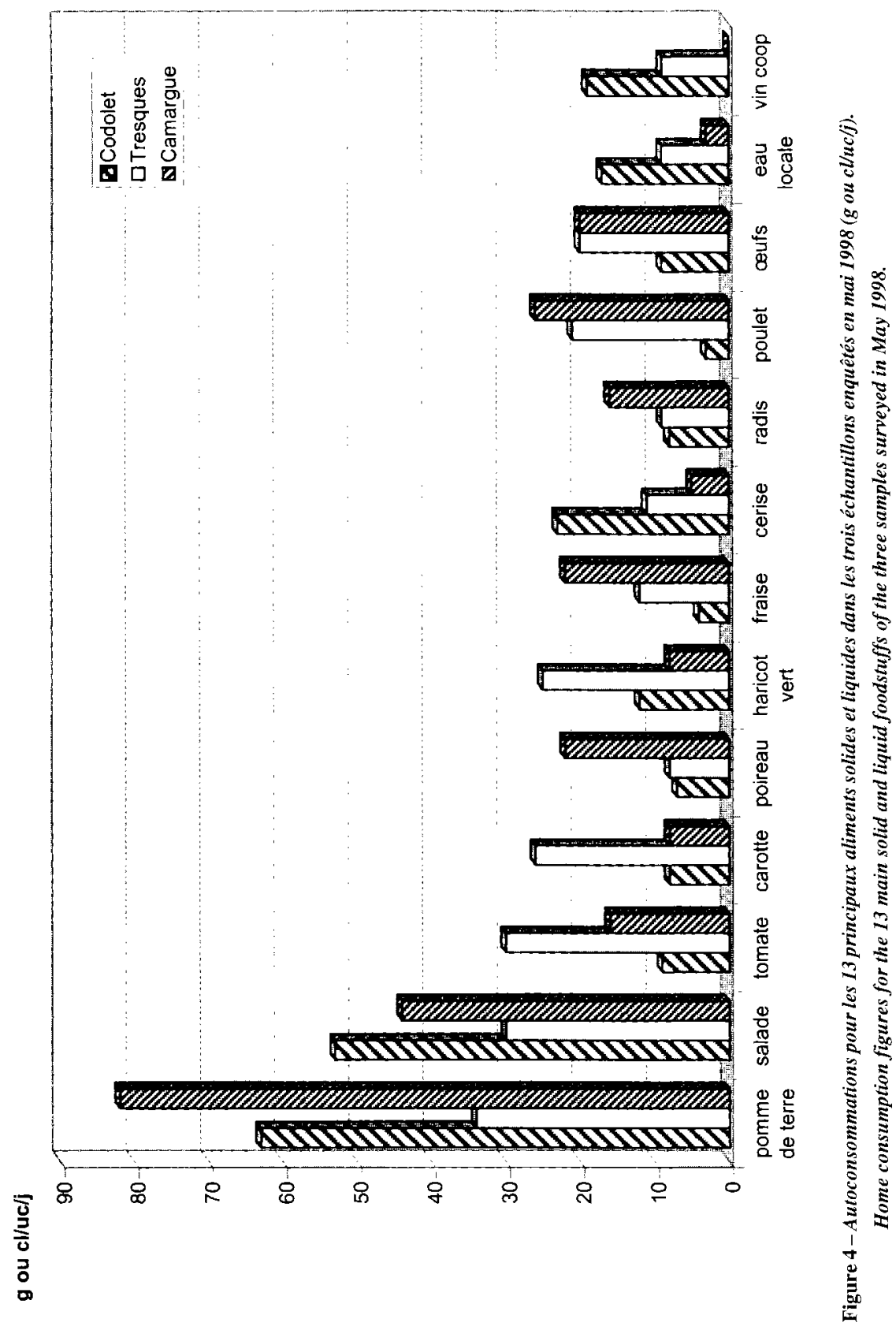


habitants de Codolet (Després, 1996). Dans ce calcul l'auteur était parti d'une hypothèse «origine locale totale» pour les aliments suivants: les légumesfeuilles, les légumes-fruits, la pomme de terre, les autres légumes-racines, les fruits, les œufs, les volailles, l'eau et le vin. La figure 5 compare, pour le village de Codolet, cette autoconsommation "supposée » dans le calcul d'impact lié à l'ingestion pour l'année 1993 avec l'autoconsommation « réelle et actualisée » obtenue dans la présente enquête.

Pour l'autoconsommation « réelle » de la présente enquête on doit apporter les précisions suivantes : les légumes-feuilles associent la salade, l'artichaut et le poireau ; les autres légumes-racines associent la carotte et le radis ; les légumesfruits associent la tomate et le haricot vert ; les fruits associent la fraise et la cerise ; les volailles ne sont constituées que du poulet ; l'eau est celle de la nappe des $10 \mathrm{~m}$ et le vin est celui de la cave coopérative.

On constate, qu'à l'exception notable du vin, toutes les autoconsommations «supposées » de l'étude de Després sont supérieures aux autoconsommations « réelles » de notre enquête. Ceci est particulièrement important pour les fruits, les œufs, les volailles et l'eau. Cette constatation indique que notre enquête pourrait être utilisée pour effectuer une réactualisation du calcul d'impact dosimétrique, lié à l'ingestion, réalisé pour l'année 1993. Dans cette hypothèse, toutes choses étant égales par ailleurs, la composante «alimentation» de cet impact s'avèrerait notablement plus faible que celle obtenue dans le calcul de l'impact de l'année 1993.

\section{Conclusion}

Cette enquête alimentaire a été réalisée à la demande de l'IPSN. Son objectif était de déterminer les habitudes alimentaires des populations, en particulier celles liées à l'autoconsommation, dans trois zones spécifiques en relation avec le site nucléaire de Marcoule. Ces trois zones sont constituées par deux villages, Codolet (le plus proche du site) et Tresques (un village «témoin ») dans le département du Gard, et par une partie de la commune d'Arles dans les Bouches-du-Rhône. Cette partie de la commune d'Arles est incluse totalement dans la zone d'extension des deux inondations survenues en octobre 1993 et en janvier 1994.

L'enquête a été effectuée en mai 1998, c'est-à-dire à une période de l'année assez particulière puisque correspondant à la période de récolte d'un certain nombre de fruits et de légumes. Soixante cinq foyers ont été enquêtés : 25 en Camargue où l'habitat est dispersé, 22 à Codolet et 18 à Tresques. 
B. DESCAMPS, F. GUILLET

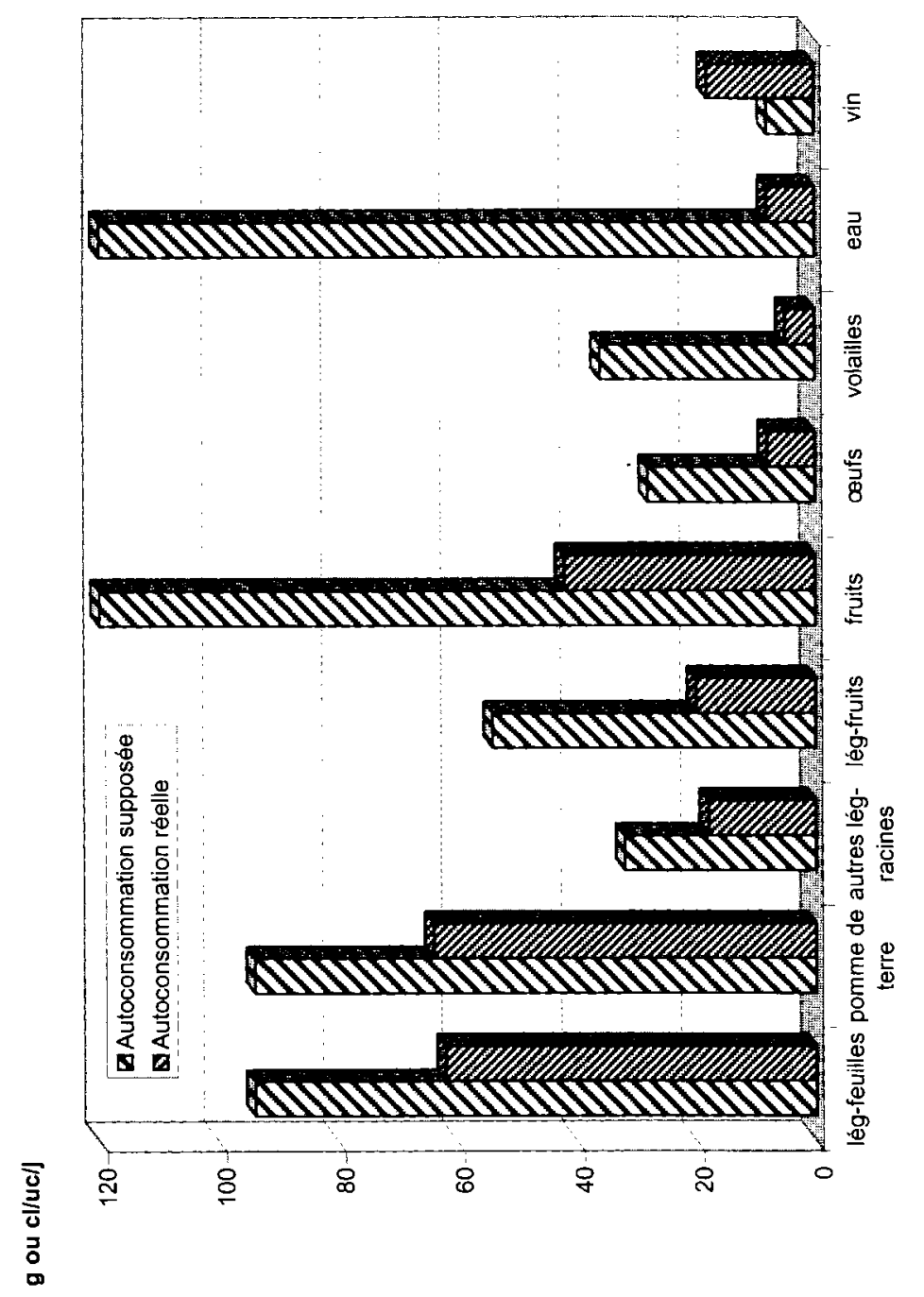

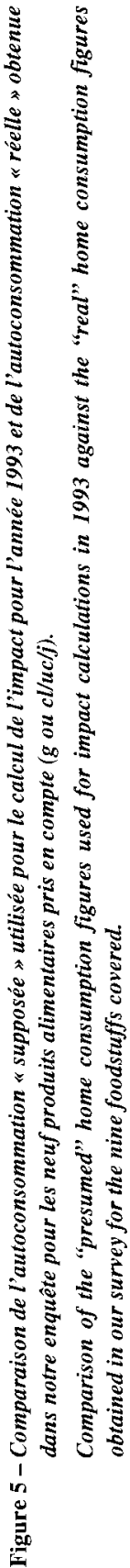


Pour ce type d'enquête il a été nécessaire de recourir à une unité fictive, l'unité de consommation (uc). Les quantités consommées et autoconsommées sont exprimées en g ou 1 par uc et par jour $\left(\mathrm{g} \mathrm{uc}^{-1} \mathrm{j}^{-1}\right.$ et $\left.\mathbf{l} \mathrm{uc}^{-1} \mathrm{j}^{-1}\right)$; on ne ferait qu'une faible approximation en les exprimant en $\mathrm{g}$ ou 1 par personne adulte et par jour, unité plus usuelle.

Les activités, sources d'autoconsommation, sont essentiellement le jardinage, l'élevage, la chasse et la pêche. Parmi les aliments autoconsommés, 13 le sont dans les trois zones, à des moyennes supérieures à $10 \mathrm{~g} \mathrm{uc}^{-1} \mathrm{j}^{-1}$ ou à $10 \mathrm{cl} \mathrm{uc}^{-1} \mathrm{j}^{-1}$. Pour les légumes et les fruits, 9 aliments sont concernés ; il s'agit par ordre décroissant $\left(\mathrm{g} \mathrm{uc}^{-1} \mathrm{j}^{-1}\right)$ : pomme de terre (62), salade (42), tomate (18), carotte (16), poireau, haricot vert et la fraise (13), cerise et radis (11). La carotte, la pomme de terre et le poireau sont plus autoconsommés en Camargue ; c'est aussi le cas pour la tomate et le haricot vert à Tresques et pour la fraise et la cerise à Codolet. Pour les produits d'origine animale seuls les cufs et le poulet sont concernés, à une valeur moyenne de $15 \mathrm{~g} \mathrm{uc}^{-1} \mathrm{j}^{-1}$. Pour les liquides, la consommation d'origine locale concerne seulement le vin et l'eau, à des moyennes de l'ordre de $0,1 \mathrm{l} \mathrm{uc}^{-1} \mathrm{j}^{-1}$. L'enquête INCA réalisée en 1999 (AFSSA/CREDOC/DGAL, 1999) ne peut être utilisée comme base de comparaison puisqu'elle ne fournit pas de données sur l'autoconsommation, d'une part, et qu'elle sépare les données relatives à la consommation des adultes de plus de 13 ans et des enfants d'age compris entre 3 et 13 ans, d'autre part. On notera toutefois que certains éléments de comparaison indiquent une tendance : certains produits (légumes, fruits, viandes, volailles, ...) sont davantage consommés dans des zones rurales agricoles, comme dans notre enquête, que dans la France entière, comme dans l'enquête INCA.

À partir des chiffres d'autoconsommation pour les habitants de Codolet notre enquête pourrait permettre d'actualiser et de préciser l'estimation, effectuée à partir de données de 1993, de l'impact dosimétrique dû à l'ingestion lié au fonctionnement du site de Marcoule. Cet impact dosimétrique actualisé serait notablement inférieur compte tenu de l'hypothèse pénalisante retenue dans cette étude de l'impact dosimétrique : toute la consommation était de l'autoconsommation.

\section{RÉFÉRENCES}

AFSSA/CREDOC/DGAL (1999) Enquête INCA, Éditions TEC \& DOC, Paris.

Cresta M., Ledermann S., Garnier A., Lombardo E., Lacourly G. (1969) Étude des consommations alimentaires des populations de onze régions de la communauté européenne en vue de la détermination des niveaux de contamination radioactive, Rapport EUR 4218 f de l'association EURATOM-CEA, 9-27.

Descamps B. (1995) Étude du marquage des feuilles de chêne de l'environnement du site de Marcoule par le tritium contenu dans les rejets atmosphériques (prélèvements d'octobre 1992), Rapport IPSN/DPEI/SERE 95/022 (P), $62 \mathrm{p}$. 


\section{B. DESCAMPS, F. GUILLET}

Descamps B., Lumia G. (1996) Étude radioécologique de l'environnement terrestre du site de Marcoule, année 1994, Rapport IPSN/DPRE/ SERE 96/019 (P), 70 p.

Descamps B. (1997) Étude radioécologique de l'environnement terrestre du site de Marcoule, année 1995, Rapport IPSN/DPRE/SERE 97/002 (P), 78 p.

Desprès A. (1996) Contribution méthodologique à l'évaluation de l'impact dosimétrique du fonctionnement du site de Marcoule, Rapport IPSN/DPHD 96-01, 49 p.

INSEE (1990a) Recensement général de la population de 1990, Gard, $220 \mathrm{p}$.

INSEE (1990b) Recensement général de la population de 1990, Bouches-du-Rhône, 155 p.

INSEE (1991) Enquête alimentaire, 267-283

Lambrechts A., Lévy F., Foulquier L., Benoit R., Jourd'heuil L., Pécout L. (1990) Suivi radioécologique de l'impact de l'usine de Marcoule sur le fleuve Rhône (1989-1990), Rapport IPSN/DPEI/SERE $90 / 07,110 \mathrm{p}$.

Lambrechts A., Lévy F., Foulquier L., Montreuil F., Jourd'heuil L., Court G., Pécout L. (1992) Suivi radioécologique de l'impact de l'usine de Marcoule sur le fleuve Rhône (1990-1991), Rapport IPSN/DPEU/SERE 92/06 (I), $88 \mathrm{p}$.

Lambrechts A., Lévy F., Foulquier L., Montreuil F., Jourd'Heuil L., Rozet M., Pécout L. (1993) Suivi radioécologique de l'impact de l'usine de Marcoule sur le fleuve Rhône (1991-1992), Rapport IPSN/DPEI/SERE 93/008 (I), 35 p.

Lambrechts A., Lévy F., Foulquier L., Montreuil F., Marchand S. (1994) Suivi radioécologique du Rhône de l'amont du site de Marcoule à l'embouchure (1992-1993), Rapport IPSN/DPEI/SERE 94/008 (I), $39 \mathrm{p}$.

Lambrechts A., Foulquier L., Pally M. (1995) Suivi radiócologique du Rhône de l'amont du site de Marcoule à l'embouchure (1993-1994), Rapport IPSN/DPEI/SERE 95/023 (P), 64 p.

Lambrechts A., Foulquier L., Pally M. (1996) Suivi radioécologique du Rhône de l'amont du site de Marcoule à l'embouchure (campagne d'octobre 1994), Rapport IPSN/DPRE/SERE 96/009 (P), $68 \mathrm{p}$.

Lumia G., Descamps B. (1995) Étude radioécologique de l'environnement terrestre du site de Marcoule, année 1993, Rapport IPSN/DPEI/SERE 95/028 (I), 67 p.

MAF (1988) Ministère de l'agriculture et de la forêt, AGRESTE, Le recensement agricole de 1988, $191 \mathrm{p}$. 